\title{
Probing the Yang-Mills vacuum with adjoint zero-modes
}

\section{Margarita García Pérez}

Instituto de Física Teórica UAM-CSIC

Universidad Autónoma de Madrid, Cantoblanco, 28049 Madrid, Spain

E-mail: margarita.garcia@uam.es

\section{Antonio González-Arroyo}

Departamento de Física Teórica and Instituto de Física Teórica UAM-CSIC

Universidad Autónoma de Madrid, Cantoblanco, 28049 Madrid, Spain

E-mail: antonio.gonzalez-arroyo@uam.es

\section{Alfonso Sastre*}

Departamento de Física Teórica and Instituto de Física Teórica UAM-CSIC

Universidad Autónoma de Madrid, Cantoblanco, 28049 Madrid, Spain

E-mail: alfonso.sastre@uam.es

For the non-perturbative analysis of the topological content of Yang-Mills theories, it is essential to disentangle long-range structures from short-range fluctuations. Some time ago, one of us proposed to use the adjoint modes of the Dirac operator for this purpose. In this talk we analyse an implementation of this idea that associates two Weyl fermionic modes in the adjoint representation to every gauge field configuration. The densities of these modes provide a filtered image of the self-dual and anti self-dual parts of the gauge action density. We present successful tests on the performance of this proposal on a set of initial gauge field configurations.

The XXVIII International Symposium on Lattice Field Theory

June 14-19,2010

Villasimius, Sardinia Italy

\footnotetext{
* Speaker.
} 


\section{Introduction}

In the past years several authors have devoted some effort to the analysis of the topological structures present in pure Yang-Mills theory on the lattice. Apart from global information such as the topological susceptibility, it is interesting to extract local one, such as the instanton size distribution. In some cases one might be able to check certain ideas and proposals relating these topological structures to chiral symmetry breaking and confinement. In this program the battlehorse is the roughness of lattice Monte-Carlo configurations, having its origin in divergent ultraviolet fluctuations. To tame this noisy background several ideas have been proposed. In particular, cooling and smearing algorithms produce smoother configurations which are assumed to preserve the long-range structure of the original one. However, these methods have been criticised since they may produce some distortions on the shape distribution of local structures. A proposed alternative is to use filtering methods based on the Dirac operator or other differential operators [1] (see for example [2] and references therein). For the case of the Dirac operator, the main idea is the relation between fermions and topology given by the Atiyah-Singer index theorem and the correlation between the gauge action density and the local density of the eigenstates of the Dirac operator. In practise, however, these methods are not totally free of ambiguities. For example, when fermions in the fundamental representation are used to reconstruct the topological charge density the ability to reproduce the topological structure depends, in a rather strong way, on the number of modes included in the reconstruction. In any case, even for noiseless configurations the shape of the filtered density does not coincide with the corresponding action density.

In this talk we analyse an alternative proposal, presented in [3], based on the use of the adjoint representation of the Dirac operator. The advantage of this method is that it is based upon a single mode and gives a perfect match for classical solutions of the equations of motion. In the next section we will give details of the method and its lattice implementation. In Section 3 we will test its performance by analysing its filtering capacity for a series of initial gauge field configuration.

\section{Description of the method}

For every gauge field configuration we will construct two associated classical Weyl fermionic fields in the adjoint representation, $\psi_{ \pm}(x)$, whose densities, $\left\|\psi_{ \pm}(x)\right\|^{2}$, correspond to a filtered image of the self-dual and anti self-dual parts of the initial gauge field action density. The Weyl spinorial fields have components $\psi_{\alpha \pm}^{a}(x)$ where $\alpha$ is a 2-component spinor index, and $a$ is the colour index taking $N^{2}-1$ values. From now on we will refer to the fields $\psi_{ \pm}$as adjoint filtering modes or AFM.

An important property that any filtering method should satisfy is that for smooth configurations the procedure must reproduce the classical structures without distortion. The main idea behind the method introduced in Ref. [3] is that for configurations that are solutions of the classical equations of motion (classical solutions) there is an optimal choice of the spinorial field in the adjoint representation that reproduces exactly the shape of the (anti)self-dual part of the gauge action density. This is obtained choosing the AFM, $\psi_{ \pm}$, as the chiral components of the supersymmetric zero-mode of the Dirac operator, defined by:

$$
\psi=\frac{1}{8} F_{\mu v}\left[\gamma_{\mu}, \gamma_{v}\right] V
$$


where $V$ is an arbitrary constant spinor. This mode has three interesting properties:

1. It is a zero-mode: $\not D \psi=0$.

2. It satisfies the reality condition, $\operatorname{Im}\left(\psi_{1 \pm}^{a}(x)\right)=0 \quad \forall a, x$.

3. Its density is exactly proportional to the (anti)self-dual part of the gauge action density.

Thus, for a classical solution, if we select a mode satisfying properties 1 and 2 , then by virtue of the third property, we obtain an optimal image of the field density structure.

For a general gauge field configuration, our method consists in finding the Weyl-spinor fields in the adjoint representation that satisfy properties 1 and 2 as much as possible. Different versions of the method follow from the relative importance given to both properties. Previous tests [3, 4] were done by looking first at the subspace of low-lying eigenmodes of the adjoint Dirac equation and then selecting the combination which best satisfies the reality condition as the AFM mode.

Here we will use an alternative more elegant proposal, also presented in Ref. [3], in which the reality condition (property 2 ) is imposed exactly. The other condition is implemented by requiring that the Weyl spinor is an eigenvector of lowest eigenvalue of $-\not D^{2}$. For a left-handed (positive chirality) spinor this becomes $-D \bar{D}$, where $D \equiv D_{\mu} \sigma_{\mu}\left(\bar{D} \equiv D_{\mu} \bar{\sigma}_{\mu}\right)$ are the Weyl operators. Actually, in the adjoint representation all eigenvalues are doubly degenerate due to euclidean $\mathrm{CP}$ invariance. Then, using any eigenvector $\psi$ and its charge conjugate $\psi_{C}=\sigma_{2} \psi^{*}$ one can form a colour vector of quaternionic matrices

$$
\left(\psi, \psi_{C}\right)=\Psi_{ \pm}^{\mu}(x) \sigma_{\mu},
$$

where $\sigma_{\mu}=(\mathbf{I},-i \vec{\tau}), \bar{\sigma}_{\mu}=(\mathbf{I}, i \vec{\tau})$ and $\tau_{i}$ are the Pauli matrices. The eigenvalue equation becomes an equation acting on quaternionic matrix fields, and the reality condition amounts to $\Psi_{ \pm}^{0}(x)=0$.

In summary, the positive chirality AFM mode is defined by the eigenvalue condition:

$$
-D \bar{D} \Psi_{+}^{i}(x) \sigma_{i}=\lambda \Psi_{+}^{i}(x) \sigma_{i}
$$

or equivalently

$$
O_{i j}^{+} \Psi_{+}^{j}(x)=\lambda \Psi_{+}^{i}(x), \text { where } O_{j k}^{+}=-D_{\mu} D_{v} \eta_{\alpha}^{\mu v} \bar{\eta}_{j}^{\alpha k},
$$

and $\eta$ and $\bar{\eta}$ are the 't Hooft symbols. The last expression can be expanded and we get

$$
O_{i j}^{+}=-\delta_{i j} D_{\mu}^{2}-\varepsilon_{i j k} \eta_{j}^{\mu v} D_{\mu} D_{v}=-\delta_{i j} D_{\mu}^{2}+i \varepsilon_{i j k}\left(E_{k}+B_{k}\right) .
$$

Notice, that the $\mathrm{O}^{+}$operator is a positive definite, real, symmetric operator, so that its eigenvalues are positive real numbers. For gauge configurations which are solutions of the classical equations of motion the lowest eigenvalue vanishes and generically is non-degenerate. The corresponding eigenvector is the supersymmetric zero mode $\Psi_{+}^{i}(x) \propto\left(E_{i}+B_{i}\right)$.

For arbitrary gauge configurations the minimum eigenvalue of $O^{+}$is non-zero. Its corresponding eigenvector is, by definition, the AFM mode. Its density provides the filtered version of the self-dual part of the action density. The usefulness of the method depends on its capacity to eliminate high frequency noise without altering the shape of smooth structures. In this talk we will present the results of our tests done on both smooth and rough configurations.

The previous formulae can be repeated for the negative chirality mode, which provides a filtered version of the anti-self-dual part of the action density. The corresponding operator is now 
replaced by

$$
O_{i j}^{-}=-\delta_{i j} D_{\mu}^{2}-i \varepsilon_{i j k}\left(E_{k}-B_{k}\right) .
$$

In order to obtain a lattice implementation of the filtering procedure it is more convenient to work with the overlap Dirac operator and construct the hermitian, positive definite matrices $H_{ \pm}^{2}=$ $P_{ \pm}\left(\gamma_{5} D_{o v}\right)^{2} P_{ \pm}$, where $P_{ \pm}$are the projectors onto the positive and negative chirality modes. The two-fold degeneracy associated with CP invariance also holds on the lattice, so that the operators can be taken to act on quaternionic vectors. Imposing the reality conditions leads to the

$$
O_{L}^{ \pm}=P_{0} H_{ \pm}^{2} P_{0},
$$

where the action of $P_{0}$ on a quaternionic field is given by $P_{0} \Psi=\Psi-\frac{1}{2} \sigma_{0} \operatorname{Tr}\left(\sigma_{0} \Psi\right)$.

The AFM modes are the eigenvectors of lowest eigenvalue of $O_{L}^{ \pm}$and can be obtained by a conjugate gradient algorithm. Details of the technique used to compute the overlap Dirac operator and other numerical aspects can be seen in Ref. [5]

\section{Testing the filtering method}

In this section, we present the results of our tests of the filtering procedure when applied to various lattice configurations. First, we apply it to an instanton configuration. Being a classical solution the method should work well, but it will allow us to quantify the finite volume and discretisation effects. Next we apply it to a series of instanton-anti-instanton (IA) pairs with varying separations. This is intended to monitor possible distortions and problems which could occur when applied to smooth configurations which are not classical solutions of the equations of motion. Finally, we will go back to the single instanton case and add stochastic noise to it. The filtering method should be able to reduce this noise and produce a neat image of the underlying smooth configuration.

\subsection{Results for Classical solutions}

The first test is done over a set of smooth, $Q=1, \mathrm{SU}(2)$ instanton configurations generated by cooling. In this case, the $\mathrm{O}^{+}$operator should have one zero-mode corresponding exactly to the supersymmetric zero-mode. As will be discussed below, discretisation and finite volume effects may shift the corresponding eigenvalue from zero. Still, we observe that the density of the lowest eigenvector of the $\mathrm{O}^{+}$operator reproduces to an excellent degree the instanton action density. Fitting both the action and the AFM mode densities to the continuum formula, we extract instanton positions and sizes that differ at most in $\Delta X=0.01 a$, and in $\Delta \rho=0.05 \rho$, respectively.

In contrast to the situation in the continuum, the lowest eigenvalue of $O_{L}^{ \pm}$is different from zero. This is due to discretisation errors and finite volume effects. The latter arise for the case of periodic boundary conditions (PBC), because $Q=1$ classical solutions do not exist on a periodic torus [6]. This is, however, not the case if twisted boundary conditions (TBC) are used instead.

To explore both effects we have generated a large set of $\mathrm{SU}(2)$ instanton configurations with varying sizes for both PBC and TBC. Fig. 1a displays the lowest eigenvalue $\left(\lambda_{1}\right)$ of the $O^{+}$operator as a function of the inverse instanton size squared $a^{2} / \rho^{2}$. As expected, $\lambda_{1}$ approaches zero in the continuum limit as $a^{2} / \rho^{4}$, with a coefficient of approximately $8 \cdot 10^{-4}$. 


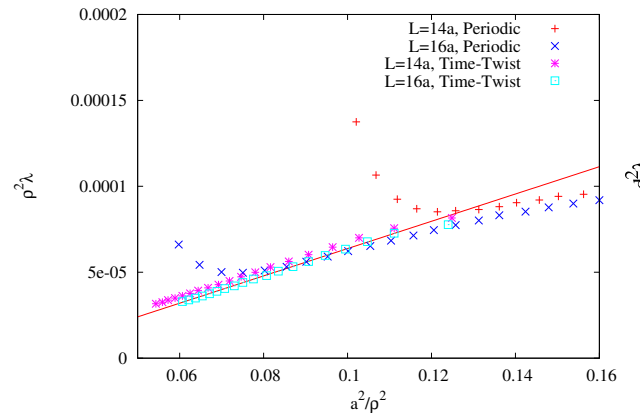

(a)

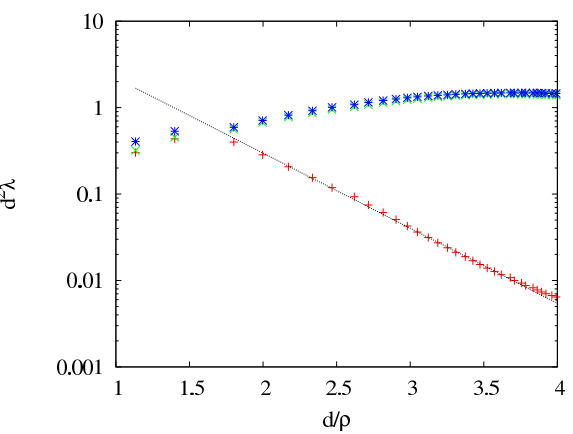

(b)

Figure 1: (a) The lowest eigenvalue of the $O^{+}$operator, on a size $\rho$ instanton background, is displayed as a function of $a^{2} / \rho^{2}$ for two different lattice sizes and boundary conditions. The finitevolume effects become sizable for periodic $\mathrm{BC}$ and $L<4 \rho$. They are negligible for time-twisted $\mathrm{BC}(k=(1,1,1), m=(0,0,0))$. (b) The three lowest eigenvalues of the $O^{+}$operator, on an IA pair background, are displayed in terms of the IA distance $(d)$. Level crossing appears at $d \sim 1.8 \rho$.

In what concerns finite-volume effects, a clear distinction can be observed between periodic and twisted boundary conditions, as expected. In the twisted case, classical solutions exist for all torus sizes and we observe no corrections to the lowest eigenvalue other that those associated to discretisation effects. For periodic BC, however, we observe a large deviation from the expected behaviour for $L \lesssim 4 \rho$.

\subsection{Smooth non-classical configurations}

According to our proposal, even for non-classical configurations the lowest eigenvector of the $O^{+}\left(O^{-}\right)$operator should provide the filtered action densities of the self-dual (anti-self-dual) part of the gauge field. Since only for classical solutions there is an exact supersymmetric zeromode, it is not guaranteed that the filtered image of non-classical configurations has no distortions. In order to test this, we have analysed a set of instanton-anti-instanton (IA) configurations with varying separation. The set is generated by cooling an initial well-separated pair and monitoring the different stages of the IA annihilation process. Fig. 2 displays one snapshot corresponding to IA distance $d=3.5 \rho$. We compare the AFM densities with the self-dual and anti-self-dual parts of the action density. The agreement is excellent and, as expected, the $O^{ \pm}$lowest eigenvectors are only sensitive to objects with the appropriate chirality. In order to obtain a more quantitative comparison, we extract the size parameter of the (anti-) instanton by fitting both the AFM and action densities. Both determinations differ by $5 \%$ at most as long as the IA separation is larger than $2 \rho$.

Although the Dirac operator does not have zero-modes on IA backgrounds, we expect the lowest eigenvalue in each chiral sector to go to zero as the I-A separation is increased. To show that this is indeed the case, we display in Fig. $1 \mathrm{~b}$ the three lowest eigenvalues of the $O^{+}$operator versus the IA separation. The smallest one is well described by an exponentially decreasing function of the IA separation as $d^{2} \lambda_{1}=16 \exp (-2 d / \rho)$. For distances $d \sim 2 \rho$ we observe a level crossing in the spectrum of the $O^{ \pm}$operators. For even smaller separations the lowest mode no longer reproduces 


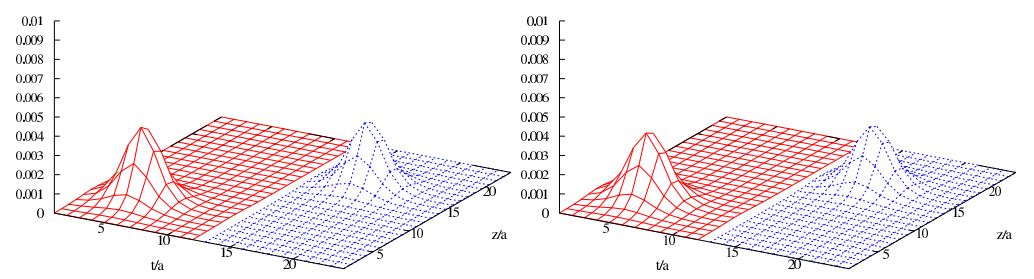

Figure 2: For an instanton-anti-instanton pair, at separation $d=3.5 \rho$, we display 2-d slices of: (Left) the self-dual (red) and the anti-self-dual (blue) parts of the action density. (Right) the AFM density corresponding to the positive (red) and the negative (blue) chiralities.

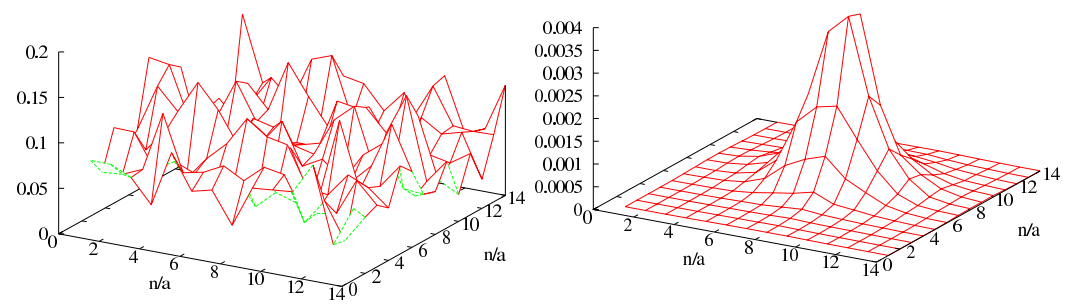

Figure 3: Comparison of the gauge action density (left) and the AFM density (right) for a configuration generated by applying ten heat-bath sweeps $(\beta=4)$ to a smooth instanton configuration.

the IA gauge action density. Hence, we conclude that the $O^{ \pm}$operators can identify the components of the IA pair as long as $d>2 \rho$.

\subsection{Configurations with stochastic noise}

The most important test, prior to its application to Monte Carlo configurations, is to show that the procedure does indeed filter out high frequency noise from the starting configuration, exhibiting its long-range structures. For this purpose, we began by generating several smooth instanton configurations and added random noise to them. The way this was done was by applying to the configuration a small number of heat bath updates corresponding to a Wilson action with different values of $\beta$. The small number of updates and the large values of $\beta$ guarantee that the instanton is not destroyed in the process, but considerable noise is added. The results presented here correspond to an initial instanton of size $\rho=3.4 a$, on a periodic/twisted lattice of size $14^{4}$, to which ten heat bath sweeps (with $\beta=30,20,8,7,6,5$, and 4 ) have been applied. The process is repeated with ten different initial random seeds giving rise to a heated instanton ensemble for each $\beta$ value and BC. A characteristic example is shown in Fig. 3, where we display the AFM and the gauge action densities for one rough configuration with $\beta=4$ and $\mathrm{PBC}$. While the action density dramatically roughens under heating, the AFM density is practically insensitive to the high frequency modes. The same is observed in the twisted case and for all the $\beta$ values analysed. This is a good proof of the extraordinary filtering capacity of our method

The ability of the method to recover the initial instanton structure is related to the fact that, despite the eigenvalue becoming non-zero after the addition of noise, there seems to be no level crossing and the AFM is always cleanly separated. This is clearly seen in Fig. 4 where we display the four lowest eigenvalues of the $O^{+}$operator as a function of $\beta^{-1}$, for periodic (left), and twisted (right) boundary conditions. Notice that not only is there no level crossing, but also the gap seems 

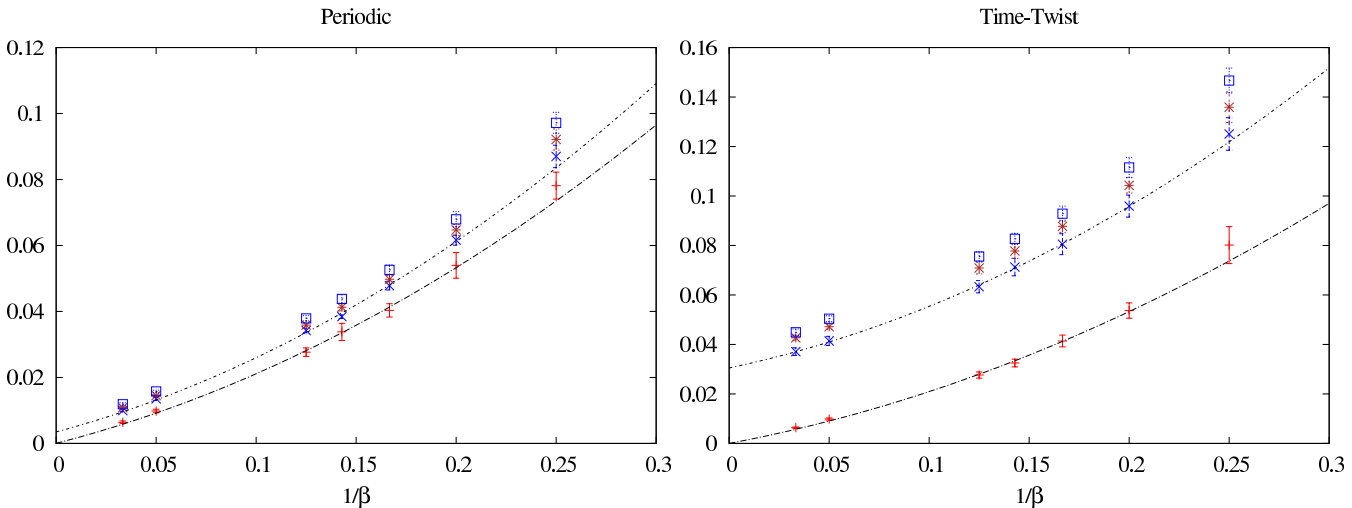

Figure 4: We display the four lowest eigenvalues of the $O^{+}$operator for the ensemble of heated instanton configurations described in section 3.3. For each value of $\beta$ we plot the eigenvalues averaged over the ensemble of heated configurations, with error bars corresponding to the variance of the sample. The continuum lines correspond to a second order degree polynomial fit in $\beta^{-1}$.

to remain constant when the size of the noise (controlled by $\beta^{-1}$ ) increases. The data for the lowest (AFM) and first excited eigenvalues of $O^{ \pm}$are well fitted by a second degree polynomial in $\beta^{-1}$, which is the form predicted by perturbation theory around the instanton configuration. The constant term is fixed to the value obtained for the instanton before heating (which for the ground state is determined by finite-volume and finite lattice spacing errors). The remaining coefficients turn to be very similar for both eigenvalues and boundary conditions.

\section{Conclusions}

In this talk we have analysed the results of the filtering method proposed in Ref. [3] when applied to different lattice configurations, some smooth and some rough. The results are quite encouraging and point out to the main difficulties one might encounter when applying the method to thermalised Monte Carlo configurations. Additional details and tests can be found in Ref. [5, 7].

\section{References}

[1] F. Bruckmann, E. -M. Ilgenfritz, Phys. Rev. D72 (2005) 114502. [hep-lat/0509020].

[2] E. -M. Ilgenfritz, K. Koller, Y. Koma et al., Phys. Rev. D76 (2007) 034506. [arXiv:0705.0018 [hep-lat]].

[3] A. Gonzalez-Arroyo and R. Kirchner, JHEP 0601 (2006) 029 [arXiv:hep-lat/0507036].

[4] M. Garcia Perez, A. Gonzalez-Arroyo and A. Sastre, PoS LAT2007 (2007) 328 [arXiv:0710.0455 [hep-lat]].

[5] Alfonso Sastre. PhD Thesis: Application of the Dirac operator in the adjoint representation to Yang-Mills theories. September 2010, Universidad Autónoma de Madrid.

[6] P. J. Braam and P. van Baal, Commun. Math. Phys. 122 (1989) 267.

[7] M. García Pérez, A. González-Arroyo and A. Sastre, work in progress. 